\title{
Post-perceptual processing during the attentional blink is modulated by inter-trial task expectancies
}

\author{
Jocelyn L. Sy ${ }^{1}$, James C. Elliott ${ }^{2,3}$ and Barry Giesbrecht ${ }^{2,3} *$ \\ 1 Department of Psychology, Vanderbilt University, Nashville, TN, USA \\ ${ }^{2}$ Department of Psychological and Brain Sciences, University of California, Santa Barbara, Santa Barbara, CA, USA \\ ${ }^{3}$ Institute for Collaborative Biotechnologies, University of California, Santa Barbara, Santa Barbara, CA, USA
}

\section{Edited by:}

Simone Vossel, Wellcome Trust Centre for Neuroimaging, University College London, UK

\section{Reviewed by:}

Roberto Dell'Acqua, University of Padova, Italy

Theodore Zanto, University of California at San Francisco, USA

\section{*Correspondence:}

Barry Giesbrecht, Department of Psychological and Brain Sciences, University of California, Santa Barbara, Santa Barbara, CA 93106, USA e-mail: barry.giesbrecht@psych. ucsb.edu
The selective processing of goal-relevant information depends on an attention system that can flexibly adapt to changing task demands and expectations. Evidence from visual search tasks indicates that the perceptual selectivity of attention increases when the bottom-up demands of the task increase and when the expectations about task demands engendered by trial history are violated. Evidence from studies of the attentional blink (AB), which measures the temporal dynamics of attention, also indicates that perceptual selectivity during the $A B$ is increased if the bottom-up task demands are increased. The present work tested whether expectations about task demands engendered by trial history also modulate perceptual selectivity during the $A B$. Two experiments tested the extent to which inter-trial switches in task demands reduced post-perceptual processing of targets presented during the $A B$. Experiment 1 indexed post-perceptual processing using the event-related potential (ERP) technique to isolate the context sensitive N400 ERP component evoked by words presented during the AB. Experiment 2 indexed post-perceptual processing using behavioral performance to determine the extent to which personal names survive the $A B$. The results of both experiments revealed that both electrophysiological (Exp. 1) and behavioral (Exp. 2) indices of post-perceptual processing were attenuated when consecutive trials differed in terms of their perceptual demands. The results are consistent with the notion that the selectivity of attention during the $A B$ is modulated not only by within-trial task demands, but also can be flexibly determined by trial-by-trial expectations.

Keywords: selective attention, event-related potentials, attentional blink, expectancy

\section{INTRODUCTION}

Human selective attention is often characterized as being flexible and dynamic, continually adapting to the information processing demands imposed by the external world and our internal goals and expectations (e.g., Corbetta and Shulman, 2002; Kastner and Pinsk, 2004; Vogel et al., 2005; Ristic and Giesbrecht, 2011; Franconeri etal., 2013). The flexibility of selective attention has been investigated by measuring the processing of stimuli that compete for attentional resources using behavioral or neuroimaging methods (e.g., Yantis and Johnston, 1990; Lavie and Tsal, 1994; Vogel et al., 2005). Demonstrations of the flexibility of attention come from studies showing that selective information processing is not fixed at either early or late stages of representation, but rather is sensitive to task demands. For instance, when attentional selectivity is measured by the behavioral interference caused by information presented at task-irrelevant spatial locations during visual search, both task demands and expectations influence the flexibility of attention. Specifically, increasing the bottom-up task demands by increasing the perceptual similarity between visual search targets and distractors can reduce the behavioral interference caused by task-irrelevant stimuli, suggesting that increasing the bottom-up task demands increases the perceptual selectivity of attention (e.g., Lavie and Cox, 1997).
Other studies have demonstrated that the selectivity of attention during visual search is also modulated by expectations generated by trial-by-trial task dependencies. For example, during visual search tasks in which difficulty varies from trial-to-trial, when the difficulty on $\operatorname{trial}_{n}$ and trial ${ }_{n-1}$ are different (switch trials) the amount of interference caused by stimuli presented at task-irrelevant locations can be reduced compared to when the search difficulty on consecutive trials is the same (repeat trials, e.g., Theeuwes et al., 2004). Together these studies, as well as other similar behavioral and neuroimaging evidence (e.g., Yantis and Johnston, 1990; Handy etal., 2001; Yi et al., 2004), support the notion that the selectivity of spatial attention is not fixed, but rather flexibly adapts to both the inherent difficulty of the task as well as one's expectations about the task.

The flexibility of attention has not only been observed in spatial visual search tasks, but also in studies designed to measure the temporal dynamics of attention. The temporal dynamics of attention are typically investigated by examining the influence of selecting and identifying one target (T1) on the processing of a subsequent target (T2). These targets can either be presented within a rapid sequence of distractors (e.g., Raymond et al., 1992; Chun and Potter, 1995) or presented briefly and then masked 
(e.g., Duncan et al., 1994; Ward et al., 1996). Observers typically have no difficulty reporting $\mathrm{T} 1$, but $\mathrm{T} 2$ detection and/or identification is impaired when it is presented within 200-500 ms of T1 (e.g., Raymond etal., 1992). This impairment is known as the attentional blink $(\mathrm{AB})$ and it is thought to represent the temporal dynamics of selection and consolidation processes (for recent reviews, see Dux and Marois, 2009; Martens and Wyble, 2010). Classic behavioral and electrophysiological studies of the $\mathrm{AB}$ have demonstrated that despite the severe impairment in $\mathrm{T} 2$ performance, semantic information about $\mathrm{T} 2$ survives the $\mathrm{AB}$ and that items presented during the $\mathrm{AB}$ can prime subsequent targets (e.g., Luck et al., 1996; Maki et al., 1997; Shapiro et al., 1997; Vogel et al., 1998; Rolke et al., 2001; Dux and Marois, 2008). Based on this evidence, theoretical accounts of the $A B$ typically assume that semantic processing is preserved during the $\mathrm{AB}$ and that the impairment in $\mathrm{T} 2$ performance occurs because of a post-perceptual failure of attention (e.g., Chun and Potter, 1995; Raymond etal., 1995; Olivers and Meeter, 2008).

In contrast to the studies showing spared semantic processing during the $\mathrm{AB}$, more recent studies have demonstrated that semantic information about T2 does not always survive the AB. For instance, Vachon and Jolicoeur (2011) and Vachon et al. (2007) have reported both behavioral and electrophysiological evidence that semantic processing within the $A B$ can be suppressed when there is a task-switch between $\mathrm{T} 1$ and $\mathrm{T} 2$. The reduction in semantic processing presumably occurs because the reconfiguration of the attentional-set from one task to the other is a resourcedemanding process that interferes with the perceptual processing of T2 (Vachon et al., 2007; Vachon and Jolicoeur, 2011). Similarly, Giesbrecht et al. $(2007,2009)$ have used both electrophysiological and behavioral approaches to demonstrate that increasing $\mathrm{T} 1$ task load can suppress the extent to which semantic and high priority information (e.g., personal names) can survive the $\mathrm{AB}$.

While the evidence from the $\mathrm{AB}$ showing reduced postperceptual processing (i.e., increased selectivity) with increasing task demands parallels the results of the visual search tasks showing reduced flanker interference and increased perceptual selectivity with increased perceptual load, there is a critical difference: in the studies of the $A B$, the selectivity of attention is measured by postperceptual processing of a task-relevant stimulus; whereas, in the visual search task selectivity is measured by the post-perceptual processing and subsequent interference caused by task-irrelevant stimuli. However, recent behavioral evidence has revealed that, much like in the visual search tasks described above, increasing T1-task load can reduce the interference caused by task-irrelevant flankers presented simultaneously with $\mathrm{T} 2$ during the $\mathrm{AB}$ (Elliott and Giesbrecht, 2010). Thus, when one considers the evidence together, the data are consistent with the notion that the perceptual demands of the T1 task can modulate the selectivity of attention within the $\mathrm{AB}$, when it is measured by the postperceptual processing of task-relevant information and when it is measured by the post-perceptual processing of task-irrelevant information.

The recent empirical evidence in the literature is consistent with the notion that the selectivity of attention during the $A B$ is flexible and modulated by the T1 task demands. However, it is unclear whether the temporal dynamics of attention are modulated by expectancies generated by inter-trial dependencies of T1 task demands. To clarify this issue, we tested whether the expectancies engendered by task-demand dependencies between trials modulate post-perceptual processing during the $\mathrm{AB}$. In two experiments, participants were presented with two masked targets displayed in rapid succession. In both experiments, the first target (T1) was a flanker-type stimulus consisting of a single arrow flanked by pairs of arrows pointing either in the same direction (congruent, e.g., $>>>>>$ ) or in different directions (incongruent, e.g., $<<><<$ ). We refer to the congruent and incongruent conditions as low and high $\mathrm{T} 1$ load, respectively (Giesbrecht et al., 2007, 2009). Unlike previous studies that have used blocked T1 load conditions to demonstrate the effects load on post-perceptual processing of information presented during the $\mathrm{AB}$ (i.e., Giesbrecht et al., 2007, 2009), in the present experiment the two types of T1 load trials were randomly intermixed within experimental blocks. The random intermixing of trials allowed us to investigate the effects of inter-trial dependencies on post-perceptual processing during the $\mathrm{AB}$ by permitting the analysis of the data as a function of whether the T1-load on a given trial was the same as the previous trial (i.e., a T1repeat trial) or was different than the previous trial (i.e., a T1-switch trial). In Experiment 1, post-perceptual processing during the $\mathrm{AB}$ was indexed by measuring the context sensitive N400 event-related potential (ERP) evoked by T2. In Experiment 2, post-perceptual processing was indexed by measuring the extent to which personal names survive the $\mathrm{AB}$. Based on studies of spatial attention (Theeuwes et al., 2004) and previous studies of the AB (Giesbrecht et al., 2007, 2009; Vachon et al., 2007; Elliott and Giesbrecht, 2010; Vachon and Jolicoeur, 2011), we predicted that the additional demands required on T1-switch trials should decrease post-perceptual processing during the $\mathrm{AB}$, relative to $\mathrm{T} 1$-repeat trials. Consistent with this prediction, we observed that T1-switches in load resulted in less semantic processing during the $\mathrm{AB}$ in both experiments.

\section{EXPERIMENT 1 \\ RATIONALE}

The purpose of Experiment 1 was to test if expectancies generated by inter-trial T1 task dependencies modulate the processing and availability of semantic information presented during the AB. To do so, we revisited the finding that the context-sensitive N400 ERP component survives the AB (Luck et al., 1996). A context word was presented at the beginning of each trial, followed by a masked flanker stimulus (T1) and a word (T2) that was either related or unrelated to the context word presented at the beginning of the trial. The magnitude of the context sensitive N400 ERP (e.g., Kutas and Hillyard, 1980) was quantified by computing the mean amplitude of the difference wave of unrelated-related trials between 300 and 500 ms post T2 stimulus onset (Luck et al., 1996; Vogel et al., 1998). Using a similar task in which high and low T1 load were presented in different blocks of trials, we previously demonstrated that the N400 evoked by T2 was not modulated by the $\mathrm{AB}$ when $\mathrm{T} 1$ load was low, but was completely suppressed during the $\mathrm{AB}$ when $\mathrm{T} 1$ load was high (Giesbrecht et al., 2007). The key issue in the present work is whether trial-by-trial 
dependencies generated when T1 load is mixed within a block of trials alters this pattern. Specifically, if semantic processing of T2 is not constrained by expectancies engendered by intertrial T1 task dependencies, then an N400 should be observed in all conditions. However, if the attentional demand imposed by inter-trial T1-switches modulates the extent to which semantic processing occurs, then the magnitude of the N400 should be reduced during the $\mathrm{AB}$ under switch compared to repeated conditions.

\section{MATERIALS AND METHODS \\ Participants}

Twelve undergraduates from the University of California, Santa Barbara (UCSB) provided informed consent and were paid $\$ 10 /$ hour for their participation (mean age $=19 ; 9$ female). The UCSB Human Subjects Committee approved all procedures.

\section{Apparatus and stimuli}

Stimulus presentation was controlled using custom scripts written for MATLAB (Mathworks, Inc., Boston, MA, USA) and the Psychophysics Toolbox (Brainard, 1997). T1 stimuli were black and consisted of a central arrow $\left(0.4^{\circ} \times 0.4^{\circ}\right)$ centered between two pairs of arrows $\left(0.4^{\circ} \times 1.1^{\circ}\right)$. The distance between adjacent arrows was $0.15^{\circ}$. The complete target stimulus subtended $0.4^{\circ} \times 2.6^{\circ}$. The context word presented at the beginning of the trial and the T2 word were black and white, respectively. Both were presented in uppercase 32-point Arial font. Each character subtended approximately $0.4^{\circ} \times 0.4^{\circ}$. T1 and T2 masks were strings of black numbers and uppercase letters the same length as the respective target. All stimuli were presented on a neutral gray background and viewed on a 19 -inch color monitor from a distance of $125 \mathrm{~cm}$.

\section{Procedure}

Each trial began with a random fixation interval (500-1000 ms), followed by the context word $(1000 \mathrm{~ms})$. After the context word there was a second random delay (750-1250 ms), followed by the presentation of T1 $(53.3 \mathrm{~ms})$ and the T1 mask $(53.3 \mathrm{~ms}$; T1-mask ISI $=53.3 \mathrm{~ms}$ ). After the temporal lag (either 320 or $920 \mathrm{~ms}$ ) lapsed, T2 was presented (40 ms) and then masked (40 ms; T2mask ISI $=40 \mathrm{~ms})$. After a third random delay $(750-1250 \mathrm{~ms})$ subjects were prompted to indicate their responses for $\mathrm{T} 1$ and $\mathrm{T} 2$. Subjects were instructed to read the context word presented at the beginning of the trial, identify the direction of the T1 central arrow (left or right) and determine whether T2 was related or unrelated to the context word. All responses were unspeeded and typed into the keyboard. After the responses were recorded, fixation returned to the screen and the participant started the next trial when ready. A sample trial sequence is shown in Figure 1.

\section{Design}

There were four independent variables: T1 load, T1 inter-trial dependency, T2-relationship, and T1-T2 lag. T1 load was manipulated by the direction of the flankers relative to the central arrow and was either congruent (i.e., $>>>>>$ or $<<<<<$ ) or incongruent (i.e., $<<><<$ or $>><>>$ ). Because the different T1 load conditions were intermixed, each trial could be categorized as T1-repeat trial (when T1-load on $\operatorname{trial}_{n}$ was the same as

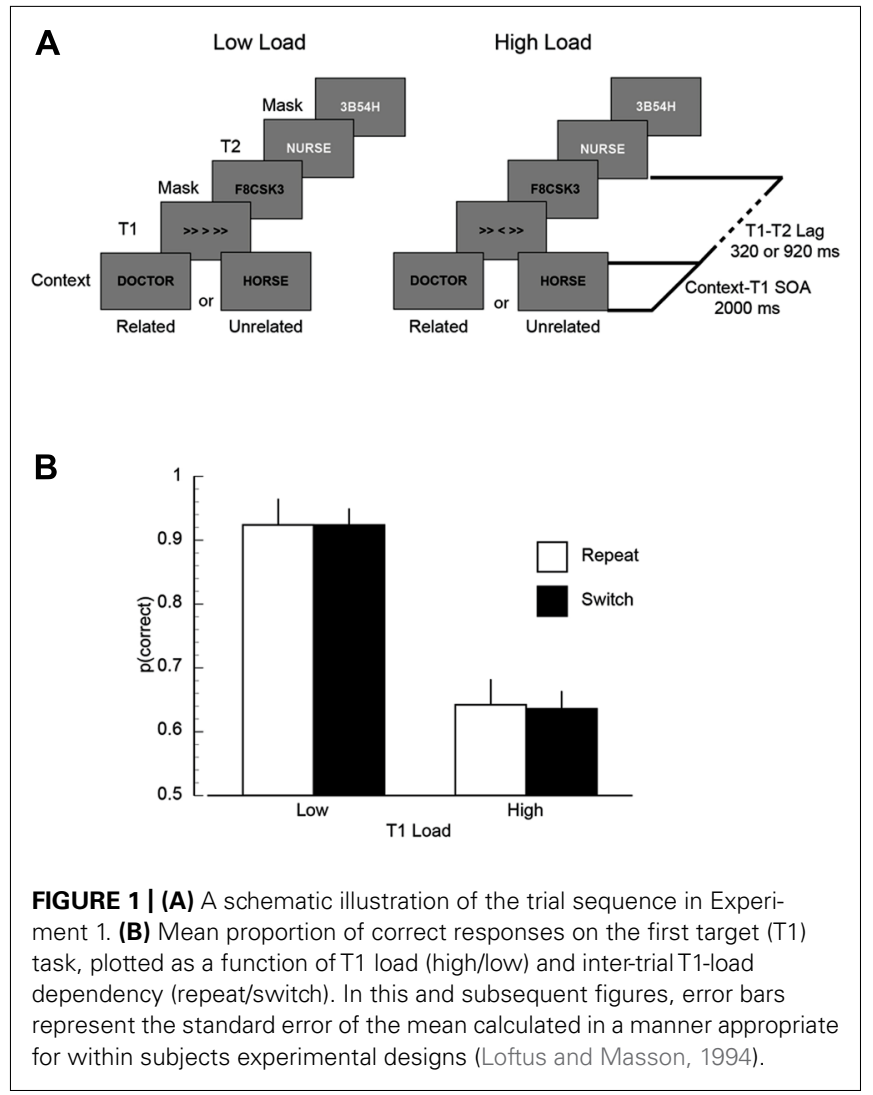

trial $_{n-1}$ ) or T1-switch trial (when T1-load on trial $_{n}$, was different from trial $_{n-1}$ ). T2-relationship specified the semantic association, either related or unrelated, between $\mathrm{T} 2$ and the context word. The specific words were compiled from previously published studies and norms (Postman and Keppel, 1970; Giesbrecht et al., 2004, 2007) and consisted of 300 related word pairs. Each word pair was randomly assigned to each of the load conditions, under the constraint that across subjects each pair was assigned to each of the load conditions an equal number of times. Unrelated word lists were created by randomly shuffling the related word pairs (Giesbrecht and Kingstone, 2004; Smallwood et al., 2011). T1-T2 lag was the temporal interval between the onsets of T1 and T2 and it was either 320 or 920 ms. T2-relationship and T1-T2 lag conditions were randomly intermixed within each block. There were 600 total trials ( 75 trials in each condition) that were divided into 10 blocks (five for each load condition) of 60 trials. Prior to the experimental trials, subjects were given 10 practice trials.

\section{Recording and analysis}

Electroencephalographic (EEG) activity was recorded at $256 \mathrm{~Hz}$ from $32 \mathrm{Ag} / \mathrm{AgCl}$ sintered electrodes mounted in an elastic cap and placed according to the International 10/20 System. The horizontal and vertical electrooculograms (EOG) were recorded from electrodes placed $1 \mathrm{~cm}$ lateral to the external canthi (left and right) and above and below each eye, respectively. The data were re-referenced offline to the average of the signal recorded from electrodes placed on the left and right mastoids and then band-pass filtered $(0.1-30 \mathrm{~Hz})$. Trials containing ocular artifacts 
(blinks and eye movements) detected by EOG amplitudes exceeding $\pm 50 \mathrm{mV}$ or by visual inspection were excluded from the analysis. The average percentage of trials that were rejected was $6.9 \%$ (range $1.3-15.2 \%$ ).

The average ERP waveforms in all conditions were computed time-locked to the onset of T2 and included a $200 \mathrm{~ms}$ prestimulus baseline and $600 \mathrm{~ms}$ poststimulus interval. The N400 was isolated by subtracting the resulting ERP waveforms on related trials from the ERP waveforms on unrelated trials. It is important to note that for a given subject, lag, and load condition the T2 word was exactly the same (only the context word was different), therefore any modulations observed in the resulting difference wave cannot be attributed to physical stimulus differences. The magnitude of the $\mathrm{N} 400$ was quantified as the mean amplitude of the difference waves over the 300-500 ms post-T2 time window. N400 measurements were obtained from frontal, central, and parietal electrodes (F3, Fz, F4, C3, Cz, C4, P3, Pz, P4, Luck et al., 1996; Vogel et al., 1998; Giesbrecht et al., 2007). As with previous studies, the mean amplitudes included both T2 correct and T2 incorrect trials (Luck et al., 1996; Vogel et al., 1998; Giesbrecht et al., 2007). The inclusion of both correct and incorrect trials should increase the likelihood that an N400 will be observed during the AB because semantic access is more likely to occur on T2 correct trials. Thus, any observed reduction in the magnitude of the $\mathrm{N} 400$ during the AB is likely to be an underestimate of the true reduction of semantic processing. Unless mentioned otherwise, within-subjects ANOVAs were used for all statistical analyses, and the $p$-values were adjusted in accordance with the Greenhouse-Geisser epsilon value.

\section{RESULTS}

\section{Behavior}

T1 accuracy. Mean proportion of correct T1 responses are plotted as a function of T1 load (low/high) and inter-trial dependency (repeat/switch) in Figure 1B. Overall mean performance was 0.78 $(\mathrm{SEM}=0.035)$. There was a significant effect of T1 load, such that performance was lower when T1 load was high $(M=0.64$, $\mathrm{SEM}=0.068)$ relative to when $\mathrm{T} 1$ load was low $(M=0.92$, $\mathrm{SEM}=0.021 ; F(1,11)=15.58, p<0.003$, MSE $=0.062)$. Neither the main effect of inter-trial dependency nor the load $\mathrm{x}$ inter-trial dependency interaction were significant (both $F$ 's $<1$ ).

T2 accuracy. Mean proportion of correct T2 responses are plotted as a function of T1 load, inter-trial dependency, and lag in Figure 2A. Overall performance was lower at short lags compared to long lags $(F(1,11)=9.81, p<0.02$, MSE $=0.013)$. While visual inspection of Figure $2 \mathrm{~A}$ suggests that there is an interaction between inter-trial dependency and lag, such that at the short lags performance on switch trials was lower than repeat trials, this interaction was not significant $(F(1,11)=2.16, p=0.17$, $\mathrm{MSE}=0.013$ ). No other effects were statistically significant.

$A B$ magnitude. Two analyses were performed using $A B$ magnitude as an index of the severity of the performance decrement caused by the T1 load and trial dependency manipulations. AB magnitude was computed by subtracting each individual's performance at the short lag (320 ms) from an optimal performance baseline (Jackson and Raymond, 2006; Giesbrecht et al., 2009). In the present experiment, the performance baseline for all conditions was the accuracy in the $920 \mathrm{~ms}$ lag, low load-repeat
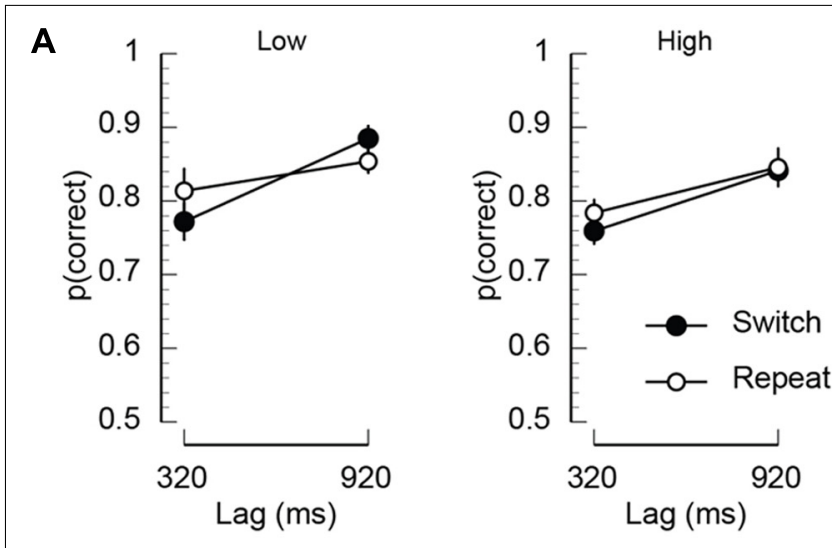

B

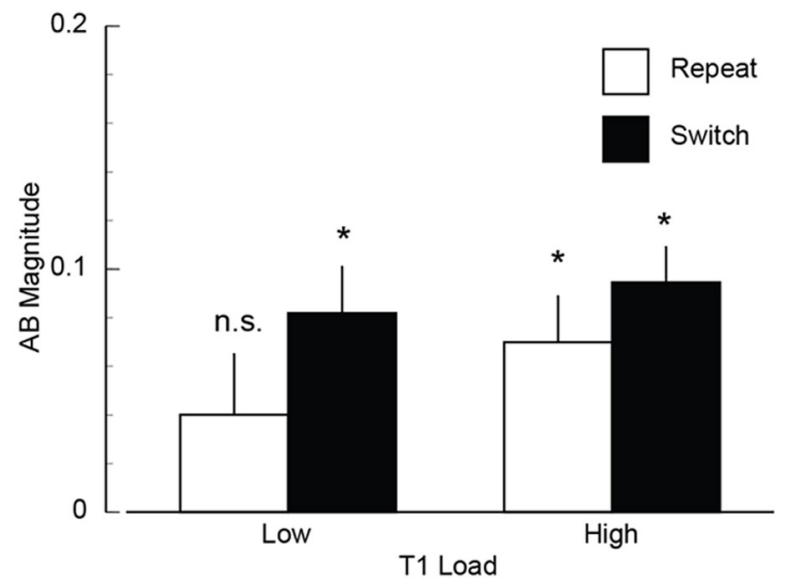

FIGURE 2 | Mean task 2 behavioral performance in Experiment 1. (A) Mean proportion of correct responses on the second target (T2) task, plotted as a function of T1 load (high/low), T1-T2 lag (320/920 ms), and inter-trial T1-load dependency (repeat/switch). (B) Mean AB magnitude plotted as a function of T1 load and inter-trial T1-load dependency. Asterisks indicate that $A B$ magnitude was significantly different than zero at $p<0.05$, FDR-corrected for multiple comparisons.

condition (i.e., the condition in which T2 accuracy should be optimal). It was appropriate to select this data point to serve as the optimal performance baseline for all conditions because the T2 stimuli were exactly the same in all conditions. It is important to note that because $\mathrm{AB}$ magnitude was computed relative to a single estimate of optimal performance (i.e., $920 \mathrm{~ms}$ lag, low load-repeat condition) instead of relative to a within condition estimate of optimal performance (e.g., the $920 \mathrm{~ms}$ lag within each condition), the ANOVA on $\mathrm{AB}$ magnitude was not redundant with the ANOVA on T2 accuracy including lag as a factor reported in the preceding paragraph. Using this metric of $\mathrm{AB}$ magnitude, the first analysis tested whether the severity of the $\mathrm{AB}$ was modulated by trial dependency and load using a repeated measures ANOVA. The results of this analysis revealed a trend for an effect of inter-trial dependency $(F(1,11)=3.40$, $p<0.1$ ), but no other significant effects. While the ANOVA 
using this metric of $A B$ magnitude as the dependent measure can indicate whether the severity of the $\mathrm{AB}$ is modulated by the experimental factors, it does not indicate the presence of an $A B$ within a specific condition. Thus, the second analysis tested for the presence of an $A B$ within each condition. To identify the presence of the $\mathrm{AB}$, one-sample $t$-tests were performed, testing whether the $A B$ magnitude in each condition was significantly different than zero (i.e., no $\mathrm{AB}$ ). $\mathrm{A}$ false discovery rate correction (FDR; Benjamin and Hochberg, 1995) was applied to correct for multiple comparisons $(p<0.05)$. The results of this analysis are shown in Figure 2B. The key finding of this analysis was that $\mathrm{AB}$ magnitude was significantly different than zero in all conditions (FDR-corrected $p$ 's $<0.006$ ), except for the repeat low load condition (FDR-corrected $p>0.28$ ).

\section{Electrophysiology}

The ERP results are summarized in Figure 3. The mean N400 difference waves measured at central electrodes $(\mathrm{C} 3 / \mathrm{Cz} / \mathrm{C} 4)$ are shown in Figure 3A as a function of inter-trial dependency, lag and time. The scalp topography during the N400 time window is shown in Figure 3B. The mean amplitude at all electrodes included in the analysis is plotted as a function of inter-trial dependency and lag in Figure 3C. Finally, the N400 mean amplitude is plotted as a function of inter-trial dependency, load, and lag for left, midline, and right electrodes in Figure 3D. The mean amplitudes were entered into a repeated measures ANOVA that included T1-load, inter-trial dependency, lag, anterior-posterior electrode position (frontal, central, parietal), and left-right electrode position (left, midline, right) as factors. The key finding that emerged from this
A
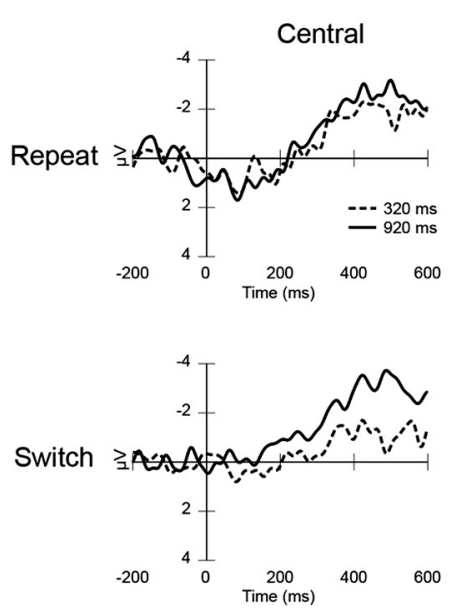

C

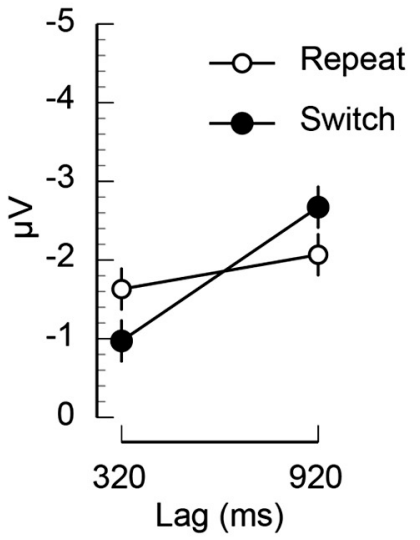

FIGURE 3 | Electrophysiological results from Experiment 2. (A) Mean unrelated-related differences waves illustrating the N400 measured at central electrodes (average at electrodes C3/Cz/C4). (B) Scalp topography of the N400 mean amplitude computed over the 300-500 ms time window. (C) Mean N400 amplitude plotted as a function of lag (320/920 ms) and inter-trial dependency. The mean amplitude was

B

D
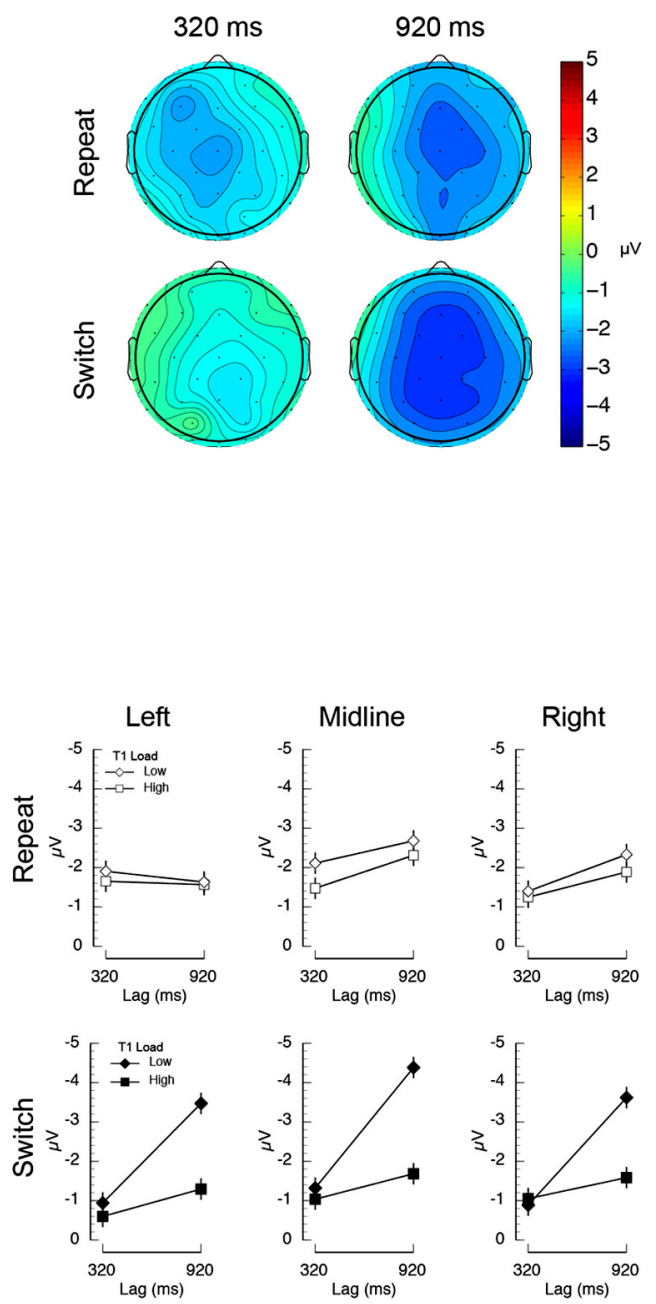

computed over the 300-500 ms post-T2 time window and averaged across all electrode sites included in the analysis (see Materials and Methods). (D) Mean N400 amplitude measured at left (F3/C3/P3), midline ( $\mathrm{Fz} / \mathrm{Cz} / \mathrm{Pz})$, and right electrodes (F4/C4/P4) plotted as a function of inter-trial dependency (repeat/switch), lag (320/920 ms), and T1 load (low/high). 
analysis was a significant interaction between inter-trial dependency and $\operatorname{lag}(F(1,11)=5.29, p<0.05, \mathrm{MSE}=16.32)$. Inspection of Figure $3 \mathrm{C}$ suggests that this interaction is being driven by the fact that the N400 is not modulated by lag on repeat trials, but is on switch trials. Post-hoc t-tests confirmed this interpretation by revealing that there was no effect of lag on T1-repeat trials $(t(11)=1.07, p>0.30)$, but the N400 was significantly smaller at the $320 \mathrm{~ms}$ lag than the $920 \mathrm{~ms} \operatorname{lag}(t(11)=2.93, p<0.02)$ on T1switch trials. This interaction is clearly visible not only in the mean amplitude data (Figure 3C), but also in the waveforms and scalp topographies (Figures 3A,B), all of which show a robust N400 on T1-repeat trials both inside and outside the $A B$, but a reduced $N 400$ on T1-switch trials during the $\mathrm{AB}$. There was also a three-way interaction between inter-trial dependency, lag, and electrode left-right position $(F(2,22)=5.34, p<0.014$, MSE $=0.788)$. This interaction (plotted with the additional factor of load in Figure 3D), was such that the inter-trial dependency $\times$ lag interaction (i.e., an effect of lag on switch trials, but not on repeat trials) was stronger at left electrode sites than midline and right electrode sites. Interestingly, while there is suggestive visual evidence that the effect of switching from high to low load had a greater impact on the N400 at short temporal lags than switching from low to high load, the three-way interaction was not significant $(F(1,11)=2.92, p>0.12$, $\mathrm{MSE}=22.51)$. The remaining main effects and interactions were also not statistically significant.

Visual inspection of the difference ERP waveforms plotted in Figure 3A suggests that the baseline of the $920 \mathrm{~ms}$ lag waveform on repeat trials is generally more positive than the corresponding condition on switch trials. To assess the extent to which this apparent modulation in the baseline is contributing to the inter-trial dependency $\times$ lag interaction, we ran a control analysis using a finer-grained pre-stimulus interval $(50 \mathrm{~ms})$. The resulting rebaselined difference waves and mean amplitudes are shown in Figure 4. While the overall inter-trial dependency $\times$ lag interaction failed to reach significance, the inter-trial dependency $\times$ lag $\times$ electrode left-right position was significant $(F(2,22)=4.06, p<0.04$, MSE $=1.69)$. As in the original analysis, and as can be clearly observed in the mean amplitudes shown in Figure 4B, this interaction was such that the inter-trial dependency $x$ lag interaction was robust over left electrodes. In contrast, at midline and right electrodes, the primary modulator of the N400 was temporal lag. This control analysis suggests that the inter-trial dependency $\times$ lag interaction is not solely being driven by differences in the prestimulus baseline, but rather is being driven by changes that are mediated by the interaction between trial-by-trial expectancies about task demands and the attentional demands caused by the $\mathrm{AB}$.

\section{SUMMARY}

The key finding in Experiment 1 was that the magnitude of the $\mathrm{N} 400$ was attenuated during the $\mathrm{AB}$ on T1-switch trials, but not on T1-repeat trials. This finding suggests that post-perceptual processing during the $\mathrm{AB}$ was modulated by the inter-trial taskdemand expectancies and that the violation of this expectancy on T1-switch trials served to increase the selectivity of attention compared to when this expectancy was not violated. Interestingly, while there was an inter-trial dependency $x$ lag interaction there was not an interaction between dependency, load, and lag. In other

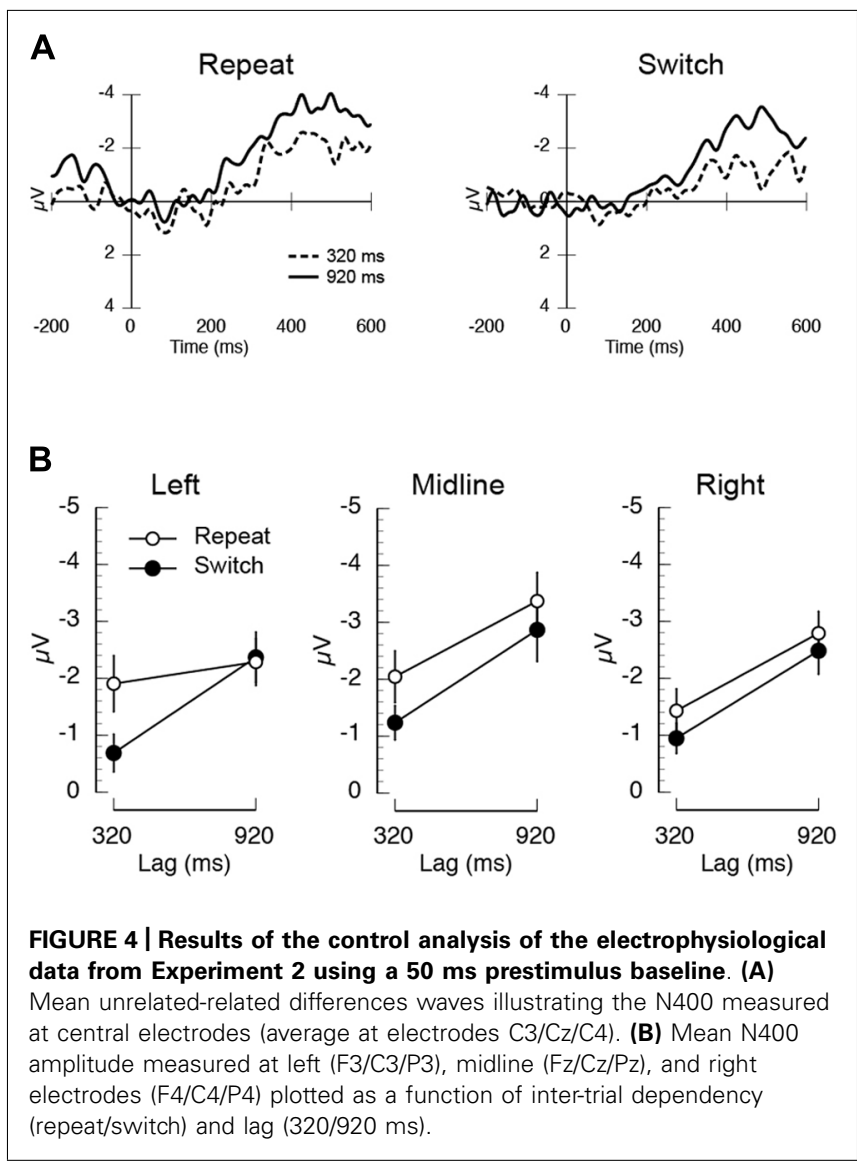

words, the dependency $\times$ lag interaction described above, was not modulated by load. This is interesting because it suggests that the previously reported effect of load on the $\mathrm{N} 400$, which serves to completely suppress the N400 during the AB (Giesbrecht et al., 2007), can be reversed by the context provided by the inter-trial dependencies.

A second result was that while inter-trial dependency did not influence T1 accuracy or overall T2 accuracy, there was suggestive evidence that dependency did affect the presence of the $\mathrm{AB}$. Specifically, there was a significant AB on T1-low load switch trials, but not on T1-low load repeat trials. Interestingly, in our previous study, an AB was observed even when T1-load was low (Giesbrecht et al., 2007). The absence of a significant $A B$ in the low-load repeat condition suggests that the expectancy generated by the inter-trial dependency causes a decrease in the difficulty of the T1 task that is sufficient to result in the absence of the $\mathrm{AB}$ on low-load trials. However, this result should be interpreted with caution because of the lack of an effect of dependency on T1 accuracy, the lack of an interaction between dependency and lag on T2 accuracy, and the lack of a significant main effect of trial dependency on $\mathrm{AB}$ magnitude.

\section{EXPERIMENT 2 RATIONALE}

To provide additional evidence that expectations engendered by trial-by-trial dependencies can modulate the selectivity of attention during the $\mathrm{AB}$, we revisited another classic 
demonstration of post-perceptual processing during the $\mathrm{AB}$ : the finding that one's own name is not subject to the $\mathrm{AB}$ (Shapiro et al., 1997). Experiment 2 tested whether the extent to which one's own name survives the $\mathrm{AB}$ is modulated by inter-trial load. There were two key manipulations. First, both T1-load and intertrial dependency were manipulated utilizing the same flanker task as in Experiment 1. However, because the behavioral effects on T1 performance and T2 performance were weak, we changed the T1 stimulus from black to white. The rationale was that the color change would make the flankers more salient and increase the likelihood that they would interfere with performance. Second, T2 was either the participant's own name (T2-own) or someone else's name (T2-other). If processing of high priority information during the $\mathrm{AB}$ is not constrained by task demands imposed by a switch trial, then there should be no $\mathrm{AB}$ for $\mathrm{T} 2$-own, but there should be an $\mathrm{AB}$ for $\mathrm{T} 2$-other, irrespective of switch in $\mathrm{T} 1$ congruency. However, if switches between trials influence the extent to which high priority information is processed, then the difference in $\mathrm{AB}$ magnitude between T2-own and T2-other conditions should be attenuated on switch trials compared to repeat trials.

\section{MATERIALS AND METHODS Participants}

Fifteen undergraduates from the University of California, Santa Barbara participated in a single 45 min session for credit in an introductory psychology class (8 female).

\section{Equipment and stimuli}

The T1 and mask stimuli, equipment, and stimulus control procedures were the same as in Experiment 1. The T2 stimuli were the subject's own name and names from the database of registered birth names available from the United States Social Security Administration (http://www.socialsecurity.gov/ OACT/babynames/). To provide a rough control for exposure to names other than one's own name, the 50 most popular male and female names were selected from the list of names that corresponded to the most common year of birth of the largely freshman introductory psychology class from which our sample was drawn (1987). All names were presented in black uppercase 32 point Arial font. Each character subtended $0.4^{\circ} \times 0.4^{\circ}$.

\section{Design}

There were two changes in the design from Experiment 1. First, T2 was either the participant's own name or another name from the list. The participant's own name appeared on one eighth of the trials. The lag between the onsets of the first and second targets ranged from 200 to $800 \mathrm{~ms}$ in steps of $120 \mathrm{~ms}$. All variables were combined factorially and randomly intermixed.

\section{Procedure}

Each trial started with a fixation cross that remained on the screen until the participant initiated the trial by pressing the space bar. After the trial was initiated, there was a random delay (500-1000 ms) followed by the presentation of T1 and its mask $($ duration $=53.3 \mathrm{~ms}$; T1-mask ISI $=53.3 \mathrm{~ms})$. After the lapsing of the temporal lag, T2 was presented $(40 \mathrm{~ms})$ and then masked (40 ms; T2-mask ISI $=40 \mathrm{~ms}$ ). On half the trials T2 was a male name and on the other half it was a female name. At the end of

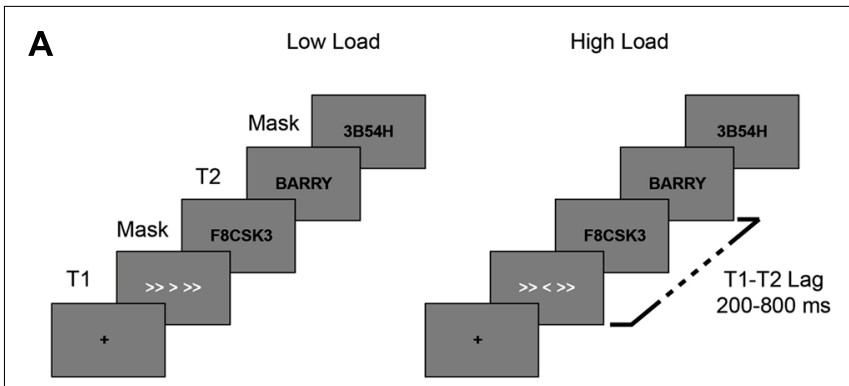

\section{B}
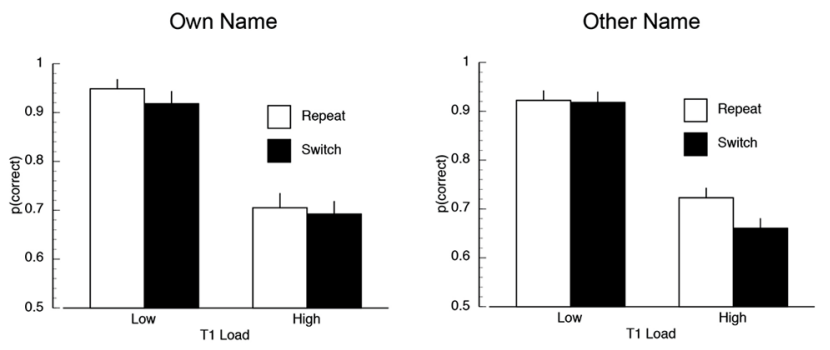

FIGURE 5 | (A) A schematic illustration of the trial sequence in Experiment 2. (B) Mean proportion of correct responses on the first target (T1) task, plotted as a function of T2 name (own/other), T1 load (high/low), and inter-trial T1-load dependency (repeat/switch).

the trial, participants were instructed to indicate the direction of the central arrow (left or right) and then whether the name was a male or a female name. All responses were unspeeded and typed into the keyboard. After the responses were indicated, the fixation cross reappeared, and the participant started the next trial when ready. An example of the trial sequence is shown in Figure 5A. Participants completed one block of 10 practice trials, followed by 10 blocks of 48 trials.

\section{RESULTS}

T1 task accuracy. The mean proportion of correct responses is plotted as a function of inter-trial dependency, load, and name in Figure 5B. There was a significant main effect of inter-trial switch where T1 accuracy was worse in switch trials $(M=0.80)$ compared to repeat trials $(M=0.83 ; F(1,14)=10.23, p<0.007$, MSE $=0.002)$. Overall performance was also higher on low load trials $(M=0.93)$ than on high load trials $(M=0.70$; $F(1,14)=33.34, p<0.001, \mathrm{MSE}=0.048)$. The only significant interaction was the inter-trial dependency $\times$ load $\times$ name interaction $(F(1,14)=4.87, p<0.05$, MSE $=0.002)$, which appeared to be driven by overall lower performance in the T1-switch high load condition when T2 was someone else's name.

T2 task accuracy. The mean proportion of correct $\mathrm{T} 2$ responses is shown in Figure 6A. Overall, inter-trial dependency modulated performance, such that overall performance was lower on switch trials than on repeat trials $(F(1,14)=5.53, p<0.04, \mathrm{MSE}=0.007)$. Mean accuracy was also lower for T2-other $(M=0.82)$ compared to T2-own $(M=0.92 ; F(1,14)=54.84, p<0.001, \mathrm{MSE}=0.035)$. There was a main effect of lag, where T2 report was worse 

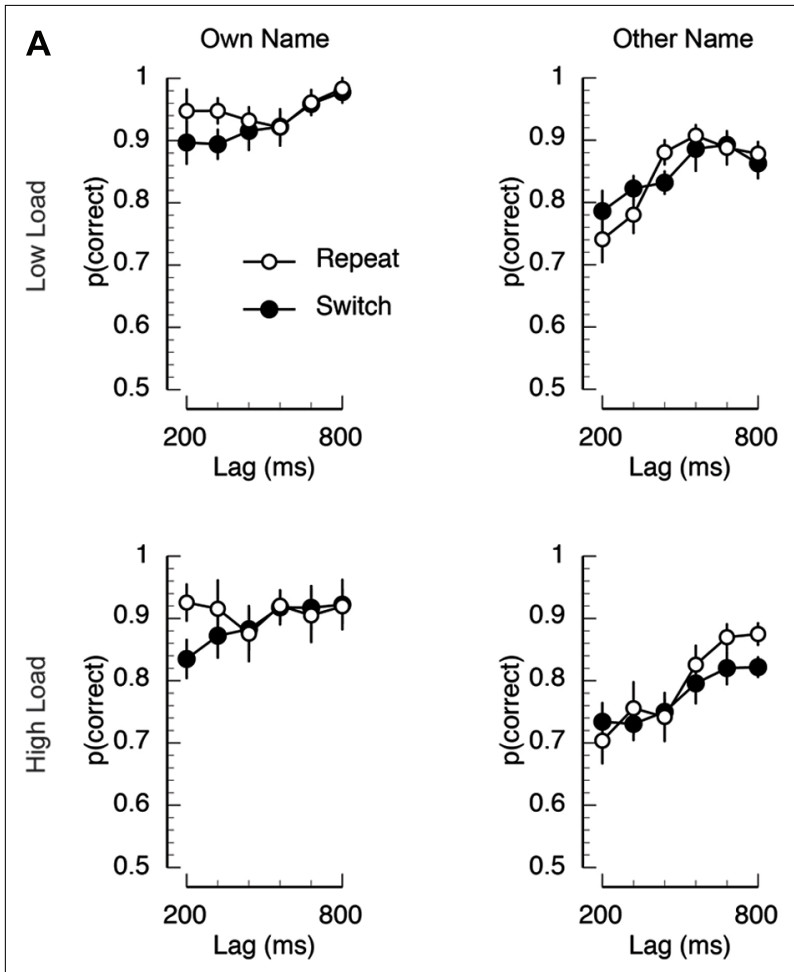

B
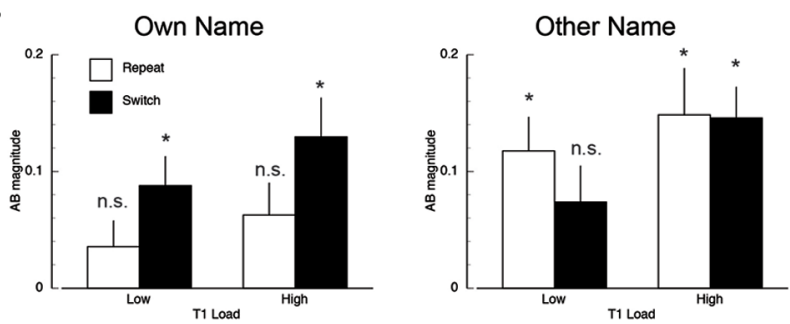

FIGURE 6 | Mean task 2 behavioral performance in Experiment 2. (A) Mean proportion of correct responses on the second target (T2) task, plotted as a function of T1 load (high/low), T2-name (own/other), T1-T2 lag (320/920 ms), and inter-trial T1-load dependency (repeat/switch). (B) Mean AB magnitude plotted as a function of T2-name (own/other), T1 load, and inter-trial T1-load dependency. Asterisks indicate that AB magnitude was significantly different than zero at $p<0.05$, FDR-corrected for multiple comparisons.

at shorter lags than longer lags $(F(5,70)=11.36, p<0.001$, MSE $=0.013)$.

There were two key interactions. First, the effect of lag was more severe for T2-other compared to T2-own $(F(5,70)=2.45$, $p<0.05$, MSE $=0.017)$. Second, and most critically, there was a three-way interaction between inter-trial dependency, name, and lag $(F(5,70)=2.46, p<0.05, \mathrm{MSE}=0.010)$. Post-hoc repeated measures ANOVAs revealed that this interaction was driven by the modulation of the name $\times$ lag interaction as a function of task dependency. Specifically, on repeat trials, there was no effect of lag for T2-own, but a large effect of lag for T2-other (name $\times$ trial: $F(5,70)=3.64, p<0.006$, MSE $=0.018)$. In contrast, on switch trials, there was an effect of lag $(F(1,14)=42.47, p<0.001$,
$\mathrm{MSE}=0.02)$ and name $(F(1,14)=17.60, p<0.002, \mathrm{MSE}=0.015)$, but no interaction $(F<1)$.

$A B$ magnitude. To further address the influence of inter-trial task dependencies on post-perceptual processing, we performed two $\mathrm{AB}$ magnitude analyses similar to those performed in Experiment 1. $\mathrm{AB}$ magnitude was computed by subtracting mean performance during the $\mathrm{AB}$ (lags 200-320 ms) from an optimal performance baseline. The baseline used in Experiment 2 was the condition in which T1-load was repeated and in which T2 was presented at the longest lag $(800 \mathrm{~ms})$. The resulting mean $\mathrm{AB}$ magnitude data are shown in Figure 6B. In the first analysis, $A B$ magnitude was entered into a repeated measures ANOVA. The key finding was that $\mathrm{AB}$ magnitude was modulated by the interaction between inter-trial dependency and name $(F(1,14)=6.73$, $p<0.022$, MSE $=0.043)$. Post-hoc tests revealed two interesting aspects to this interaction. First, in the T2-own condition, AB magnitude was significantly smaller on repeat trials than on switch trials $\left(M_{\mathrm{T} 2-\text { own,repeat }}=0.05, M_{\mathrm{T} 2-\text { own, switch }}=0.109 ; t(14)=3.04\right.$, $p<0.009)$. In contrast, in the T2-other condition there was no difference between repeat and switch trials $\left(M_{\mathrm{T} 2-\text { other,repeat }}=0.13\right.$, $\left.M_{\mathrm{T} 2-\text { other,switch }}=0.11 ; t(14)=1.25, p>0.23\right)$. Second, on repeat trials AB magnitude was significantly larger in the T2-other condition compared to the T2-own condition $\left(M_{\mathrm{T} 2-\text { other,repeat }}=0.05\right.$, $\left.M_{\mathrm{T} 2-\text { own,repeat }}=0.13 ; t(14)=2.74, p<0.02\right)$, but on switch trials there was no difference in $\mathrm{AB}$ magnitude $\left(M_{\mathrm{T} 2-\text { other,switch }}=0.11\right.$, $\left.M_{\mathrm{T} 2-\text { own,switch }}=0.109 ; t(14)=0.04, p<0.97\right)$. In the second analysis, just as in Experiment 1, the presence of the $A B$ in each condition was identified using one-sample $t$-tests (vs. zero). A FDR correction (Benjamin and Hochberg, 1995) was applied to correct for multiple comparisons $(p<0.05)$. The key finding of this analysis was that $\mathrm{AB}$ magnitude was significantly different than zero in all conditions (FDR-corrected $p$ 's $<0.02)$, except for the own name repeat condition under both low and high load (FDR-corrected $p>0.11$ ). In addition, $\mathrm{AB}$ magnitude in other name switch condition under low load was also not significantly different than zero (FDR-correct $p>0.11)$.

\section{SUMMARY}

The survival of personally meaningful information during the $\mathrm{AB}$ has been used to argue that some post-perceptual information is available during the AB (Shapiro et al., 1997). Overall performance on repeat trials replicated this previous finding showing there is no $\mathrm{AB}$ in response to one's own name, but there is an $\mathrm{AB}$ to other people's names. There were two main findings that were novel. First was the finding that expectancies engendered by inter-trial task dependencies modulated the severity of the $\mathrm{AB}$ when the second target was one's own name. Second, overall there was an AB in both T2-own name and T2-other name conditions when T1 load switched from the previous trial. Together, both the mere presence of an $\mathrm{AB}$ for one's own name on switch trials and the fact that the severity of the $\mathrm{AB}$ for one's own name can be modulated by intertrial task dependencies (i.e., $\mathrm{AB}$ magnitude was larger on switch relative to repeat trials) supports the idea that the post-perceptual processing of high priority stimuli can be attenuated during the $\mathrm{AB}$ by a violation of trial-by-trial expectancies generated during the course of one's experience with a task. One exception to this 
pattern was in the other name switch condition under low load, in which the test for the presence of the $A B$ did not reach the FDR-corrected threshold. When using an uncorrected threshold the $\mathrm{AB}$ magnitude was different than zero $(p<0.04$, uncorrected), suggesting that there may be a weak $\mathrm{AB}$ for other names on low load switch trials. A final interesting finding is that while previous work has shown that increases in T1 task demands can cause an AB for one's own name (Giesbrecht et al., 2009), the absence of an $A B$ for one's own name on repeat high load trials is suggestive evidence that the expectancies generated by inter-trial repetitions of high load are sufficient to override the effect of load on the current trial.

\section{DISCUSSION}

The purpose of the present work was to test the extent to which expectancies about task demands engendered by the trial history of T1 task load modulate post-perceptual information processing during the AB. Experiment 1 tested the magnitude of the N400 evoked by $\mathrm{T} 2$ words during the $\mathrm{AB}$ and demonstrated that when T1 task load was repeated from the previous trial, the N400 survived the $A B$. Importantly, when $T 1$ task load switched from the previous trial, the $\mathrm{N} 400$ evoked during the $\mathrm{AB}$ was attenuated relative to outside the AB. Experiment 2 tested if inter-trial dependencies influenced the extent to which personal names survive the AB. The results revealed that on T1-repeat trials one's own name survived the $A B$, but other names did not. However, on T1-switch trials, an AB was present for both one's own name and someone else's name. This suggests that inter-trial switches of T1load reduced the availability of highly salient information during the $\mathrm{AB}$.

Previous studies have shown that manipulations of task demands within a trial can attenuate post-perceptual processing during the AB (Giesbrecht et al., 2007, 2009; Vachon et al., 2007; Vachon and Jolicoeur, 2011). The novel finding in both of the present experiments is that inter-trial dependencies of task demand, induced by repetitions and switches in T1-flanker congruency between trials, attenuated the availability of semantic information during the $\mathrm{AB}$. This new finding contrasts theoretical accounts of the $\mathrm{AB}$ that propose that information presented during the $\mathrm{AB}$ is processed to a post-perceptual level despite the impairment in report (e.g., Chun and Potter, 1995; Raymond etal., 1995; Olivers and Meeter, 2008). However, the present results are consistent with the growing literature demonstrating that the failure that gives rise to the $\mathrm{AB}$ can occur either at post-perceptual and perceptual (i.e., pre-semantic) stages of processing (e.g., Giesbrecht et al., 2007, 2009; Vachon et al., 2007; Vul etal., 2008; Elliott and Giesbrecht, 2010). Importantly, these more recent findings suggest that the level at which selective attention operates during the $\mathrm{AB}$ is flexibly determined by T1-task demands (e.g., Giesbrecht et al., 2007, 2009; Vachon et al., 2007; Elliott and Giesbrecht, 2010; Vachon and Jolicoeur, 2011).

The finding that post-perceptual processing during the $A B$ is attenuated by inter-trial dependencies of task load, parallels the finding in the visual search literature showing that post-perceptual processing of task irrelevant information is also attenuated by inter-trial switches of task demands (e.g., Theeuwes et al., 2004).
These results can be explained in the context of the conflict adaptation literature that suggests that managing changes in conflict between consecutive trials is an effortful process that requires more top-down attentional control in order to resolve conflict either by an active reconfiguration of task set, or by an active inhibition of the previous task set, or both (e.g., Rogers and Monsell, 1995; Monsell, 2003; Rossi et al., 2009). However, it is important to distinguish switch costs in the traditional sense, defined by a change in stimulus-response rules, from the switch costs in the current experiments where the participants performed the identical T1 task in all trials and only the perceptual difficulty changed between trials. However, more recent work has demonstrated that perceptual switches involving changes in the number of simultaneously presented features as in the present experiments resulted in similar if not greater behavioral switch costs than when compared with more typical task-switches (cf. Ullsperger et al., 2005; Ravizza and Carter, 2008).

The availability of post-perceptual information during the $\mathrm{AB}$ when T1-congruency was repeated and the reduction of postperceptual information during the $\mathrm{AB}$ when $\mathrm{T} 1$-congruency was switched between trials can be explained with a flexible selection account of attention. Flexible selection models posit that the level of information processing at which attention selects relevant information is dependent on concurrent task demands (e.g., Yantis and Johnston, 1990; Lavie, 1995, 2005; Pashler, 1998; Lavie et al., 2004; Vogel et al., 2005). In the context of the AB, an over investment of attentional resources on $\mathrm{T} 1$ required by a highly demanding task, such as a switch in task or high T1load within a trial, may reduce the available resources available to process subsequent items presented rapidly beyond a perceptual level (Giesbrecht et al., 2007, 2009; Vachon et al., 2007; Elliott and Giesbrecht, 2010; Vachon and Jolicoeur, 2011). Effectively, the increase in T1-task demands increases the subsequent selectivity of processing, as measured by post-perceptual processing of T2. Thus, the present results support the proposal that the level of processing during the $\mathrm{AB}$ is flexible and not always fixed at a post-perceptual level and, more broadly, demonstrates that the human attention system develops expectancies about task difficulty that modulates both the spatial and temporal selectivity of attention.

\section{ACKNOWLEDGMENTS}

This research was generously supported by the Institute for Collaborative Biotechnologies through grant W911NF-09-0001 from the U.S. Army Research Office. The content of the information does not necessarily reflect the position or the policy of the Government, and no official endorsement should be inferred. The authors thank Megan Lewis and Noelle Baumann for their assistance with data collection for Experiment 2. We also thank the two reviewers for their constructive comments, including Reviewer 1 who suggested the control analysis reported in Experiment 1.

\section{AUTHOR CONTRIBUTIONS}

Barry Giesbrecht designed and programmed the experiments. Jocelyn L. Sy, James C. Elliott, and Barry Giesbrecht collected the data. Jocelyn L. Sy and Barry Giesbrecht analyzed the data. Jocelyn L. Sy, James C. Elliott, and Barry Giesbrecht wrote the manuscript. 


\section{REFERENCES}

Benjamin, Y., and Hochberg, Y. (1995). Controlling the false discovery rate: a practical and powerful approach to multiple testing. J. R. Stat. Soc. Ser. B 57, 289-300. doi:10.2307/2346101

Brainard, D. H. (1997). The psychophysics toolbox. Spat. Vis. 10, 433-436. doi: 10.1163/156856 897X00357

Chun, M. M., and Potter, M. C. (1995). A two-stage model for multiple target detection in rapid serial visual presentation. J. Exp. Psychol. Hum. Percept. Perform. 21, 109-127. doi: 10.1037/0096-1523.21.1.109

Corbetta, M., and Shulman, G. L. (2002). Control of goal-directed and stimulus-driven attention in the brain. Nat. Rev. Neurosci. 3, 201-215. doi: 10.1038/nrn755

Duncan, J., Ward, R., and Shapiro, K. (1994). Direct measurement of attentional dwell time in human vision. Nature 369, 313-315. doi: 10.1038/369313a0

Dux, P. E., and Marois, R. (2008). Distractor inhibition predicts individual differences in the attention blink. PLoS ONE 3:e3330. doi: 10.1371/journal.pone.0003330

Dux, P. E., and Marois, R. (2009). The attentional blink: a review of data and theory. Atten. Percept. Psychophys. 71, 1683-1700. doi: 10.3758/APP.71.8.1683

Elliott, J. C., and Giesbrecht, B. (2010). Perceptual load modulates the processing of distractors presented at task-irrelevant locations during the attentional blink. Attent. Percept. Psychophys. 72, 2106-2114. doi: 10.3758/APP.72.8.2106

Franconeri, S. L., Alvarez, G. A., and Cavanagh, P. (2013). Flexible cognitive resources: competitive content maps for attention and memory. Trends Cogn. Sci. 17, 134-141. doi: 10.1016/j.tics.2013.01.010

Giesbrecht, B., Camblin, C. C., and Swaab, T. Y. (2004). Separable effects of semantic priming and imageability on word processing in human cortex. Cereb. Cortex 14, 521-529. doi: 10.1093/cercor/bhh014

Giesbrecht, B., and Kingstone, A. (2004). Right hemisphere involvement in the attentional blink: evidence from a split-brain patient. Brain Cogn. 55, 303-306. doi: 10.1016/j.bandc.2004.02.026

Giesbrecht, B., Sy, J. L., and Elliott, J. E. (2007). Electrophysiological evidence for both perceptual and post-perceptual selection during the attentional blink. J. Cogn. Neurosci. 19, 2005-2018. doi: 10.1162/jocn.2007.19.12.2005
Giesbrecht, B., Sy, J. L., and Lewis, M. K. (2009). Personal names do not always survive the attentional blink: behavioral evidence for a flexible locus of selection. Vis. Res. 49, 1378 1388. doi: 10.1016/j.visres.2008 02.013

Handy, T. C., Soltani, M., and Mangun, G. R. (2001). Perceptual load and visuocortical processing: event-related potentials reveal sensory-level selection. Psychol. Sci. 12, 213-218. doi: 10.1111/14679280.00338

Jackson, M. C., and Raymond, J. E. (2006). The role of attention and familiarity in face identification. Percept. Psychophys. 68, 543-557. doi: 10.3758/BF03208757

Kastner, S., and Pinsk, M. A. (2004). Visual attention as a multilevel selection process. Cogn. Affect. Behav. Neurosci. 4, 483-500. doi: 10.3758/CABN.4.4.483

Kutas, M., and Hillyard, S. A. (1980). Reading senseless sentences: brain potentials reflect semantic incongruity. Science 207, 203-205. doi: 10.1126/science.7350657

Lavie, N. (1995). Perceptual load as a necessary condition for selective attention. J. Exp. Psychol. Hum. Percept. Perform. 23, 451-468. doi: 10.1037/0096-1523.21.3.451

Lavie, N. (2005). Distracted and confused?: selective attention under load. Trends Cogn. Sci. 9, 75-82. doi: 10.1016/j.tics.2004.12.004

Lavie, N., and Cox, S. (1997). On the efficiency of attentional selection: efficient visual search results in inefficient rejection of distraction. Psychol. Sci. 8, 395-398. doi: 10.1111/j.14679280.1997.tb00432.x

Lavie, N., Hirst, A., de Fockert, J. W., and Viding, E. (2004). Load theory of selective attention and $\operatorname{cog}$ nitive control. J. Exp. Psychol. Gen. 133, 339-354. doi: 10.1037/00963445.133.3.339

Lavie, N., and Tsal, Y. (1994). Perceptual load as a major determinant of the locus of selection in visual attention. Percept. Psychophys. 56, 183-197. doi: 10.3758/BF03213897

Loftus, G. R., and Masson, M. E. J. (1994). Using confidence intervals in within-subjects designs. Psychon. Bull. Rev. 1, 476-490. doi: 10.3758/BF03210951

Luck, S. J., Vogel, E. K., and Shapiro, K. L. (1996). Word meanings can be accessed but not reported during the attentional blink. Nature 383, 616-618. doi: 10.1038/ $383616 \mathrm{a} 0$

Maki, W. S., Frigen, K., and Paulson, K. (1997). Associative priming by targets and distractors during rapid serial visual presentation: does word meaning survive the attentional blink? J. Exp. Psychol. Hum. Percept. Perform. 23, 1014 1034. doi: 10.1037/0096-1523.23. 4.1014

Martens, S., and Wyble, B. (2010). The attentional blink: past, present, and future of a blind spot in perceptual awareness. Neurosci. Biobehav. Rev. 34, 947-957. doi: 10.1016/ j.neubiorev.2009.12.005

Monsell, S. (2003). Task switching. Trends Cogn. Sci. 7, 134-140. doi: 10.1016/S1364-6613(03)00028-7

Olivers, C. N. L., and Meeter, M. (2008). A boost and bounce theory of temporal attention. Psychol. Rev. 115, 836-863. doi: 10.1037/a0013395

Pashler, H. (1998). The Psychology of Attention. Cambridge, MA: MIT Press.

Postman, L. J., and Keppel, G. (1970). Norms of Word Association. New York: Academic Press.

Ravizza, S. M., and Carter, C. S. (2008). Shifting set about task switching: behavioral and neural evidence for distinct forms of cognitive flexibility. Neuropsychologia 46, 2924-2935. doi: 10.1016/j.neuropsychologia.2008.06 006

Raymond, J. E., Shapiro, K. L., and Arnell, K. M. (1992). Temporary suppression of visual processing in an RSVP task: an attentional blink? J. Exp. Psychol. Hum. Percept. Perform. 18, 849-860. doi: 10.1037/00961523.18.3.849

Raymond, J. E., Shapiro, K. L., and Arnell, K. M. (1995). Similarity determines the attentional blink. J. Exp. Psychol. Hum. Percept. Perform. 21, 653-662. doi: 10.1037/00961523.21.3.653

Ristic, J., and Giesbrecht, B. (2011). Electrophysiological evidence for the spatiotemporal flexibility in the ventrolateral attention network. PLoS ONE 6:e24436. doi: 10.1371/journal.pone. 0024436

Rogers, R. D., and Monsell, S. (1995). Costs of a predictable switch between simple cognitive tasks. J. Exp. Psychol. Gen. 124, 207-231. doi: 10.1037/0096-3445.124.2.207

Rolke, B., Heil, M., Streb, J., and Henninghausen, E. (2001). Missed prime words within the attentional blink evoke an N400 semantic priming effect. Psychophysiology 38, 165-174. doi: 10.1111/1469-8986. 3820165

Rossi, A. F., Pessoa, L., Desimone, R., and Ungerleider, L. G. (2009). The prefrontal cortex and the executive control of attention. Exp. Brain Res.
192, 489-497. doi: 10.1007/s00221 008-1642-z

Shapiro, K., Driver, J., Ward, R., and Sorensen, R. E. (1997). Priming from the attentional blink: a failure to extract visual tokens but not visual types. Psychol. Sci. 8, 95100. doi: 10.1111/j.1467-9280.1997. tb00689.x

Shapiro, K. L., Caldwell, J., and Sorensen, R. E. (1997). Personal names and the attentional blink: a visual "cocktail party" effect. J. Exp. Psychol. Hum. Percept. Perform. 23, 504-514. doi: 10.1037/00961523.23.2.504

Smallwood, J., Brown, K. S., Tipper, C., Giesbrecht, B., Franklin, M. S., Mrazek, M. D., et al. (2011). Pupillometric evidence for the decoupling of attention from perceptual input during offline thought. PLoS ONE 6:e18298. doi: 10.1371/journal.pone.0018298

Theeuwes, J., Kramer, A. F., and Belopolsky, A. V. (2004). Attentional set interacts with perceptual load in visual search. Psychon. Bull. Rev. 11, 697-702. doi: 10.3758/BF03 196622

Ullsperger, M., Bylsma, L. M., and Botvinick, M. M. (2005). The conflict adaptation effect: it's not just priming. Cogn. Affect. Behav. Neurosci. 5, 467-472. doi: 10.3758/CABN.5.4.467 Vachon, F., and Jolicoeur, P. (2011). Impaired semantic processing during task-set switching: evidence from the $\mathrm{N} 400$ in rapid serial visual presentation. Psychophysiology 48, 102-111. doi: 10.1111/j.14698986.2010.01040.x

Vachon, F., Tremblay, S., and Jones, D. M. (2007). Task-set reconfiguration suspends perceptual processing: evidence from semantic priming during the attentional blink. J. Exp. Psychol. Hum. Percept. Perform. 33, 330-347. doi: 10.1037/0096-1523.33.2.330

Vogel, E. K., Luck, S. J., and Shapiro, K. L. (1998). Electrophysiological evidence for a post-perceptual locus of suppression during the attentional blink. J. Exp. Psychol. Hum. Percept. Perform. 24, 1656-1674. doi: 10.1037/0096-1523.24.6.1656

Vogel, E. K., Woodman, G. F., and Luck, S. J. (2005). Pushing around the locus of selection: evidence for the flexible-selection hypothesis. J. Cogn. Neurosci. 17, 1907-1922. doi: 10.1162/089892905775008599

Vul, E., Nieuwenstein, M., and Kanwisher, N. (2008). Temporal selection is suppressed, delayed, and diffused during the attentional blink. Psychol. Sci. 19, 55-61. doi: 10.1111/j.14679280.2008.02046.x 
Ward, R., Duncan, J., and Shapiro, K. (1996). The slow time-course of visual attention. Cogn. Psychol. 30, 79-109. doi: 10.1006/cogp.1996.0003 Yantis, S., and Johnston, J. C. (1990). On the locus of visual selection: evidence from focused attention tasks. J. Exp. Psychol. Hum. Percept. Perform. 16, 135-149. doi: 10.1037/00961523.16.1.135

Yi, D.-J., Woodman, G. F., Widdlers, D., Marois, R., and Chun, M. M.
(2004). Neural fate of ignored stimuli: dissociable effects of perceptual and working memory load. Nat. Neurosci. 7, 992-996. doi: 10.1038/ nn1294

Conflict of Interest Statement: The authors declare that the research was conducted in the absence of any commercial or financial relationships that could be construed as a potential conflict of interest.
Received: 07 June 2013; accepted: 10 September 2013; published online: 08 October 2013.

Citation: Sy JL, Elliott JC and Giesbrecht B (2013) Post-perceptual processing during the attentional blink is modulated by inter-trial task expectancies. Front. Hum. Neurosci. 7:627. doi: 10.3389/fnhum.2013. 00627

This article was submitted to the journal Frontiers in Human Neuroscience.
Copyright (c) 2013 Sy, Elliott and Giesbrecht. This is an open-access article distributed under the terms of the Creative Commons Attribution License (CC BY). The use, distribution or reproduction in other forums is permitted, provided the original author(s) or licensor are credited and that the original publication in this journal is cited, in accordance with accepted academic practice. No use, distribution or reproduction is permitted which does not comply with these terms. 